Rolle spielen, aber auch in der analytischen Zahlentheorie oder der theoretischen Physik auftauchen.

Das systematische Studium singulärer Integrale ist Anfang der fünfziger Jahre durch A. P. Calderón und A. Zygmund begonnen worden. Für eine große Klasse solcher Operatoren, zu denen auch die Hilberttransformation gehört, ist seitdem die Beschränktheit der Operatoren auf den wichtigsten Funktionenräumen nachgewiesen. Insbesondere ist die Hilberttransformation beschränkt auf $L^{p}$ für $1<p<\infty$. Beim Studium analoger Operatoren auf Lipschitz-Kurven stieß Calderón Mitte der sechziger Jahre auf die bilineare Hilberttransformation

$$
B(f, g)(x)=\operatorname{pv} \int_{\mathbb{R}} f(x-t) g(x+t) \frac{d t}{t}
$$

und stellte die Frage nach $L^{p}$-Schranken für $B$. Insbesondere vermutete er, daß $B: L^{2} \times L^{2} \mapsto L^{1}$ beschränkt sei.
Bis vor kurzem blieb selbst die Frage, ob $B$ überhaupt $L^{p}$-Abbildungseigenschaften besitzt, offen. In einer bahnbrechenden Arbeit [1] gelang es Lacey und Thiele Mitte letzten Jahres, dieses Problem in den Griff zu bekommen und die Beschränktheit der bilinearen Hilberttransformation für $\frac{1}{p_{1}}+\frac{1}{p_{1}}=\frac{1}{p}$ und $2<p_{1}, p_{2}<\infty, p<2$ zu beweisen (für den Fall $1<p_{1}, p_{2} \leq \infty, 2 / 3<p$, siehe [2]). Die von Lacey und Thiele dabei entwickelten Methoden der Phasenraumanalysis haben das Gebiet der Fourieranalysis enorm bereichert und werden sicherlich noch viele weitere Anwendungen finden.

[1] M. Lacey \& C. Thiele: $L^{p}$-estimates on the bilinear Hilbert transform for $2<p<\infty$, erscheint in Annals of Math.

[2] M. Lacey \& C. Thiele: On Calderon's conjecture, Preprint.

(D. Müller, Kiel/GMZ)

\title{
Bezeichnungen in der Mathematik
}

\section{von Gerhard Brecht}

Unter dieser Überschrift stand ein Beitrag von mir in den DMV-Mitteilungen 3/94. Er wurde ergänzt durch den Beitrag "Überarbeitung von DIN 1302“ von Herrn Oberschelp aus Kiel in den DMVMitteilungen 1/96. Durch beide Beiträge sollte um neue Mitarbeiter für die Überarbeitung der wichtigen mathematischen Norm

\section{DIN 1302}

Allgemeine mathematische Zeichen und Begriffe

geworben werden. Als Obmann dieser Aufgabe möchte ich in diesem Beitrag kurz darlegen, was sich seither getan hat und was schon erarbeitet wurde.

Im Herbst 1995 fand die konstituierende Sitzung der Aufgabe 44 (Mathematische Zeichen) des AEF im DIN statt. Auf dieser Sitzung wurde besprochen, was in den ersten Entwurf von DIN 1302 aufgenommen werden soll. Herr Dr. Wolf von der Universität - GH Essen und ich wurden beauftragt, auf der Sitzung im Herbst 1996 einen Entwurf für DIN 1302 vorzulegen. In diesen wurden folgende "Dinge“ neu aufgenommen, die in der jetzt gültigen Fassung von DIN 1302 nicht oder anders enthalten sind. In der Tabelle findet man jeweils eine Darstellung in Kurzform.

Allgemein bekannt sind die Mengen $\mathbb{N}(\operatorname{mit} 0), \mathbb{Z}, \mathbb{Q}$, $\mathbb{R}$ und $\mathbb{C}$. Das hochgestellte Zeichen $\star$ hinter jedem dieser Zeichen schließt die Null aus, z.B. $\mathbb{R}^{*}=\mathbb{R} \backslash\{0\}$.
Eine Einschränkung auf Teilbereiche jeder der aufgeführten Mengen kann in sinnvoller und verallgemeierungsfähiger Weise z.B. wie folgt vorgenommen werden: $\mathbb{R}_{>0}, \mathbb{Z}_{\leq-8}, \mathbb{Q}_{\leq 0}, \mathbb{R}_{>0}^{2}$.

Diese Art der Kennzeichnung ist relativ neu, setzt sich in der mathematischen Literatur zunehmend durch und soll daher in die neue Norm DIN 1302 aufgenommen werden. Z.B. ist $\mathbb{R}_{>0}$ sofort verständlich, was man von den auch zu findenden Bezeichnungen $\mathbb{R}^{+}, \mathbb{R}_{+}, \mathbb{R}_{+}^{*}$ und $\mathbb{R}_{++}$für diese Teilmenge von $\mathbb{R}$ nicht ohne weiteres sagen kann.

Bei den elementaren Funktionen wird jetzt auch auf die im englischen Sprachraum verwendeten Funktionsbezeichnungen $\sec x$ für $\frac{1}{\cos x}, \operatorname{cosec} x$ oder kurz $\csc x$ für $\frac{1}{\sin x}$, sech $x$ für $\frac{1}{\cosh x}$ und $\operatorname{cosech} x$ oder kurz $\operatorname{csch} x$ für $\frac{1}{\sinh x}$ hingewiesen.

Die Nebenwerte der Arkusfunktionen (im Reellen) werden jetzt mit $\arcsin _{k} x, \arccos _{k} x, \arctan _{k} x$ und $\operatorname{arccot}_{k} x$ bezeichnet, anders als bisher in DIN 1302, wo z.B. $\operatorname{arc}_{k} \sin x$ empfohlen wurde mit $k \in \mathbb{Z}$ und dem Hauptwert $\operatorname{arc}_{0} \sin x=\operatorname{Arcsin} x$. Der Hauptwert wird jetzt kurz mit $\arcsin x$ anstelle $\arcsin _{0} x$ oder $\operatorname{Arcsin} x$ bezeichnet.

Für den Haupt- und die Nebenwerte des komplexen Logarithmus wurde mein Vorschlag aus dem Beitrag in den DMV-Mitteilungen 3/94 übernommen.

Im neuen Abschnitt 12 werden einige mathematische 


\begin{tabular}{|c|c|c|}
\hline Nr. 1.6 & $x \doteq y$ & $y$ ist ein gerundeter Wert von $x, \pi \doteq 3,1416$ \\
\hline Nr. 3.9 & $(x)_{n}$ & $\begin{array}{l}\text { Steigende Faktorielle, auch Pochhammer-Symbol genannt. } \\
(x)_{n}=x(x+1) \ldots(x+n-1) \text {. Anstelle von }(x)_{n} \text { wird auch } x^{\bar{n}} \text { verwendet. }\end{array}$ \\
\hline Nr. 3.10 & {$[x]_{n}$} & $\begin{array}{l}\text { Fallende Faktorielle. }[x]_{n}=x(x-1) \ldots(x-n+1) \text {. Anstelle }[x]_{n} \text { wird auch } \\
x^{\underline{\underline{n}}} \text { verwendet. In der Kombinatorik und Statistik, wo die steigende Faktorielle }\end{array}$ \\
\hline Nr. 3.14 & $|x|$ & $\begin{array}{l}\text { nicht gebraucht wird, ist das Zeichen }(x)_{n} \text { für die fallende Faktorielle verbreitet. } \\
\text { größte ganze Zahl kleiner oder gleich } x,|\pi|=3,|-\pi|=-4\end{array}$ \\
\hline Nr. 3.15 & $\lceil x\rceil$ & kleinste ganze Zahl größer oder gleich $x,\lceil\pi\rceil=4,\lceil-\pi\rceil=-3$ \\
\hline Nr. 3.16 & $\operatorname{int} x$ & ganzzahliger Anteil von $x, \operatorname{int} \pi=3, \operatorname{int}(-\pi)=-3$ \\
\hline Nr. 3.17 & $\operatorname{frac} x$ & gebrochener Anteil von $x$, frac $\pi=0,14159 \ldots$, frac $(-\pi)=-0,14159$. \\
\hline Nr. 5.6 & $\mathbb{P}$ & Menge der Primzahlen \\
\hline Nr. 8.4 & $x \rightarrow a+$ & $\begin{array}{l}x \text { von rechts gegen } a \text {. Anstelle von } a+\text { schreibt man auch } a+0 \text {. } \\
\text { Analog ist } a-\text { erklärt. }\end{array}$ \\
\hline Nr. 8.5 & $f(a+)$ & $\begin{array}{l}\text { Rechtsseitiger Grenzwert von } f(x) \text { für } x \rightarrow a+\text {. } \\
\text { Anstelle } f(a+) \text { schreibt man auch } f(a+0) \text {. Analog ist } f(a-) \text { erklärt. }\end{array}$ \\
\hline Nr. 9.18 & $f_{a}^{0} f(x) d x$ & $\begin{array}{l}\text { Cauchyscher Hauptwert. Auch in Gebrauch sind die Bezeichnungen } \\
\text { HW } \int_{a}^{b} f(x) d x, \oint_{a}^{b} f(x) d x, \operatorname{pv} \int_{a}^{b} f(x) d x\end{array}$ \\
\hline Nr. 9.19 & $f_{-\infty}^{\infty} f(x) d x$ & $\begin{array}{l}\text { Cauchyscher Hauptwert. Auch in Gebrauch sind die Bezeichnungen } \\
\text { HW } \int^{\infty} f(x) d x, \stackrel{\infty}{\complement} f(x) d x \text {, pv } \int^{\infty} f(x) d x\end{array}$ \\
\hline Nr. 12.1 & $\begin{array}{l}\varepsilon(x) \\
H(x)\end{array}$ & $\begin{array}{l}\text { Einheitssprungfunktion, } \\
\text { Heavisidefunktion, }\end{array} \varepsilon(x)=\left\{\begin{array}{lll}0 & \text { für } \quad x<0, \\
1 & \text { für } \quad x>0,\end{array}\right.$ oft wird $\varepsilon(0)=\frac{1}{2}$ gesetzt. \\
\hline Nr. 12.2 & $\operatorname{rect}(x)$ & $\begin{array}{l}\text { Rechteckimpulsfunktion, rect }(x)=\left\{\begin{array}{lll}1 & \text { für } & |x|<\frac{1}{2} \\
0 & \text { für } & |x|>\frac{1}{2} \\
\text { oft wird rect }\left( \pm \frac{1}{2}\right)=\frac{1}{2} \text { gesetzt. }\end{array} \quad \text { }\right.\end{array}$ \\
\hline Nr. 12.3 & $\operatorname{tri}(x)$ & Dreieckimpulsfunktion, $\operatorname{tri}(x)=\left\{\begin{array}{ccc}1-|x| & \text { für } & |x| \leq 1 \\
0 & \text { für } & |x|>1\end{array}\right.$ \\
\hline Nr. 12.4 & $\operatorname{sinc}(x)$ & Spaltfunktion, $\operatorname{sinc}(x)=\left\{\begin{array}{cl}\frac{\sin \pi x}{\pi x} & \text { für } x \neq 0 \\
1 & \text { für } x=0\end{array}\right.$ \\
\hline Nr. 12.5 & $\operatorname{inv}(x)$ & Involut $x$, Evolventenfunktion, $\operatorname{inv}(x)=\tan x-x$ \\
\hline
\end{tabular}

Hilfsfunktionen aufgeführt, die sich aus elementaren Funktionen ganz oder stückweise zusammensetzen, in den Anwendungen sehr häufig auftreten und bis auf $\varepsilon(x)$ bisher in keiner DIN-Norm aufgeführt worden sind.

Etwas aus dem Rahmen von DIN 1302 fällt die normmäßige Festlegung $e(\varphi)=\left(\begin{array}{l}\cos \varphi \\ \sin \varphi\end{array}\right)$ für den ebenen Einheitsvektor, gewissermaßen die reelle Version von $e^{i \varphi}$. Sie wurde von der Anwenderseite gewünscht.

Wir würden uns freuen, wenn dieser Beitrag Anlaß zu konstruktiver Kritik an unserer Arbeit ist und vielleicht den einen oder anderen Leser dazu bewegt bei uns mitzuarbeiten. Die nächste Arbeitssitzung findet im September 1997 (38. Woche) in Bad Lauterberg statt. Alle derzeit gültigen DIN-Normen zum Fachgebiet Mathematik findet man im DIN-Taschenbuch 202 aus den Beuth-Verlag in Berlin, das 1994 erschienen ist.

Der zuständige Normenausschuß im DIN ist jetzt der Normenausschuß Technische Grundlagen (NATG), der kürzlich durch Zusammenschluß einiger anderer Normenausschüsse entstanden ist. Der AEF arbeitet weiter als Fachbereich A des NATG.

\section{Adresse des Autors:}

Prof. Dr. Gerhard Brecht

Eckenerweg 29

25524 Itzehoe 